\title{
STUDY OF TENECTEPLASE IN PATIENTS WITH ST ELEVATED ACUTE MYOCARDIAL INFARCTION
}

\author{
Vemula Sreenivasulu1, K. S. S. Venkateswara Rao 2
}

${ }_{1}^{1}$ Associate Professor, Department of Medicine, Government Hospital, Anantapur, Andhra Pradesh.

2 Professor, Department of Medicine, Government Hospital, Anantapur, Andhra Pradesh.

\section{BACKGROUND}

ABSTRACT

The incidence of CAD is increasing rapidly. Thrombolytic therapy revolutionised the treatment of acute STEMI. The efficacy, safety of Tenecteplase in STEMI is very much proved. The objective of this study is to study the efficacy and safety of tenecteplase in patients with ST elevated acute myocardial infarction.

\section{MATERIALS AND METHODS}

This is a retrospective observational study done in 100 patients from June 2016 to May 2017.

\section{RESULTS}

The overall rate of clinically successful thrombolysis (CST) by tenecteplase is $92 \%$. The success rate of delayed administration ( 6 hours) was $84 \% .92 \%$ had chest pain relief with a medium time of 15 minutes. ST segment resolution was observed in $94 \%$ patients. Reinfarction was observed in 3\% of patients. Bleeding excluding ICH was observed in $2 \%$ of patients. 3 patients died before discharge. No hypotension was observed with tenecteplase.

\section{CONCLUSION}

Our study further reinforced the efficacy and safety of tenecteplase in patients presenting with STEMI including high risk subgroups. Our study confirms the importance of early thrombolysis in Indian Scenario.

\section{KEYWORDS}

STEMI- Tenecteplase - Clinically Successful Thrombolysis (CST).

HOW TO CITE THIS ARTICLE: Sreenivasulu V, Rao KSSV. Study of tenecteplase in patients with ST elevated acute myocardial infarction. J. Evolution Med. Dent. Sci. 2018;7(06):705-707, DOI: 10.14260/jemds/2018/160

\section{BACKGROUND}

Coronary artery disease is the most common cardiac disorder in India. Acute Myocardial Infarction is one of its severe complications. The incidence of CAD is increasing, especially in developing countries like India. Its incidence is increased in younger patients and also in rural areas. A survey conducted in 45 rural villages in India reported $32 \%$ of all deaths were due to cardiovascular diseases.

Primary coronary intervention (PCI) is the preferred reperfusion therapy for patients presenting with acute STEMI when performed by an experienced team in India. CREATE registry reveal that the average time taken by Indian STEMI patient to get hospitalised was 300 minutes. ${ }^{1} \mathrm{~A}$ recent study done by Noorani et al showed that nothing has changed for pre-hospital delays even today. ${ }^{2}$

There are 625 active catheterisation laboratory centres in India. Approximately, 2 lakhs PCI procedures are done in India per year. So the possibility of performing primary PCI for all Indian STEMI patients is far from reality within the time frame of 120 minutes as recommended in the guidelines. Pharmacological fibrinolysis is well accepted in patients presenting early after symptoms onset, that is within 3 hours.

'Financial or Other Competing Interest': None.

Submission 27-12-2017, Peer Review 25-01-2018,

Acceptance 31-01-2018, Published 05-02-2018.

Corresponding Author:

Dr. Vemula Sreenivasulu,

D. No. 6-4-53-1,

Maruthi Nagar,

Anantapur-515001,

Andhra Pradesh.

E-mail: drsreenivasuluvemula9@gmail.com

DOI: $10.14260 /$ jemds $/ 2018 / 160$
The preferred fibrinolytic strategy includes combination of bolus tenecteplase, aspirin, clopidogrel and enoxaparin as initial therapy. Tenecteplase with multiple evidence-based advantages is the best suitable option in Indian STEMI patients in whom primary PCI cannot be performed. ${ }^{3}$

\section{MATERIALS AND METHODS}

This is a retrospective observational study. Case records of patients presenting to ICCU with chest pain, diagnosed as acute STEMI, in whom primary PCI was not feasible within 120 minutes of diagnostic ECG. Study was conducted on 100 patients in Govt. General Hospital, Anantapur from June 2016 to May 2017.

Protocol- Inj. Tenecteplase, Adjuvant Medication as per Physician's instructions.

\section{Inclusion Criteria}

1. Chest pain diagnosed as STEMI.

2. Thrombolysed with tenecteplase.

3. Recording all parameters mentioned as study data points in the patient's health record file was mandatory.

\section{Exclusion Criteria}

1. Patients with incomplete health records.

2. Contraindications for tenecteplase.

3. PCI within the previous month.

4. Previous CABG.

Table 1. Patient Features

1. Mean age 54 years.

2. M: F $1.98: 1$.

3. Hypertension $74 \%$.

4. Diabetes $49 \%$.

5. Previous CAD $26 \%$.

6. Smoking $40 \%$.

7. Hyperlipidaemia $46 \%$. 
Table 2. Infarct Location

1. Anterior $54 \%$.

2. Inferior $36 \%$.

3. Lateral $1 \%$.

4. Anteroseptal 2\%.

5. Anterolateral $2 \%$.

6. Posterior $2 \%$.

7. Right ventricle $3 \%$.

\section{Table 3. Killip Class}

1. Class $1,46 \%$.

2. Class $2,38 \%$.

3. Class $3,12 \%$.

4. Class $4,4 \%$.

\section{Table 4. Concomitant Drugs Given}

1. Aspirin $100 \%$.

2. Clopidogrel $100 \%$.

3. Atorvastatin $100 \%$.

4. Beta blockers $70 \%$.

5. Nitroglycerin $20 \%$.

6. Low molecular weight heparin $92 \%$.

7. Enalapril $60 \%$.

\section{RESULTS}

100 patients of STEMI are studied in Govt. General Hospital, Anantapur, with the above criteria. The rate of Clinically Successful Thrombolysis (CST) with tenecteplase was $92 \%$. Those who received within 3 hours of symptoms was $94 \%$. Those who received between 3 - 6 was $89 \%$ and those after 6 hours was $84 \%$.

STE resolution was observed in $94 \%$ of patients. Within 90 minutes, STE resolution was $91 \%$. Mean time for resolution of STE was 70 minutes. $92 \%$ has relief of chest pain. Mean time of chest pain relief was 58 minutes. Within 30 minutes, $35 \%$ of patients had relief of chest pain. Reinfarction occurred in $2 \%$ of patients. 3 patients died before discharge. No incidence of Hypotension was observed. The incidence of bleeding excluding ICH is $2 \%$. Incidence of stroke is $2 \%$.

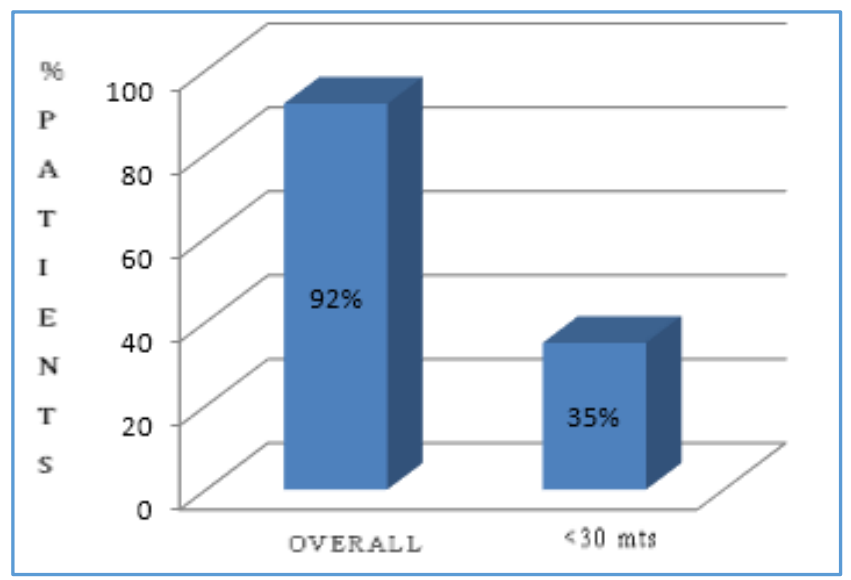

Figure 1. Chest Pain Relief
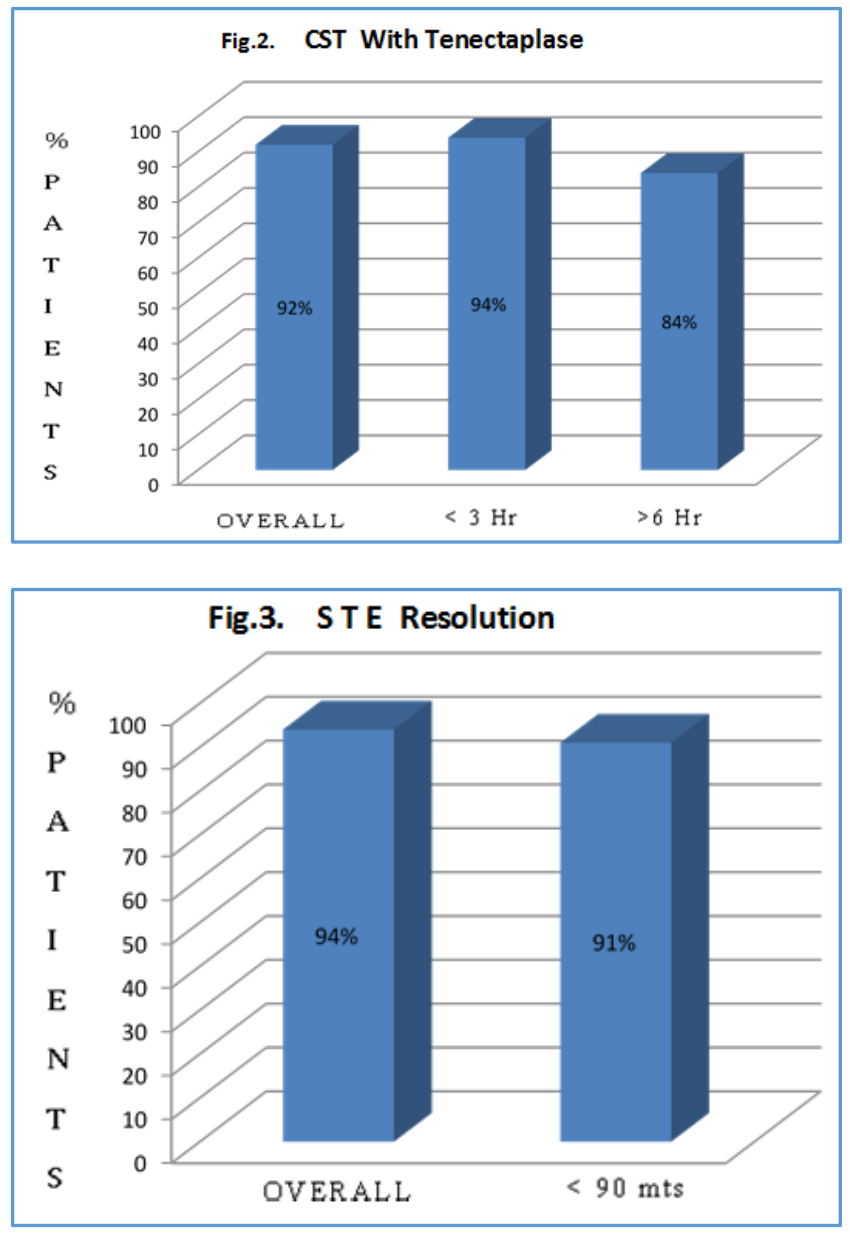

\section{DISCUSSION}

CREATE was a large prospective clinical registry of acute coronary syndrome patients in 89 large hospital centres in India. In CREATE study, the median time from symptoms to hospital in STEMI patients was 300 minutes. 38.1\% reached hospital in less than 4 hours. Study by Noorani et al, the medial total pre-hospital delay was 240 minutes. In our study, $76 \%$ of patients presented within 3 hours of chest pain.

Iyengar et al analysed the efficacy and safety of tenecteplase in 15,222 patients with STEMI. ${ }^{4}$ Overall, $95.43 \%$ of patients had clinically successful thrombolysis with higher success rate $(96.54 \%)$ in patients treated within 3 hours, success rate of $85.38 \%$ in those presented in 6 hours. STREAM trial demonstrated that patients presented within 3 hours of symptoms onset and fibrinolysed had better outcome $^{5}$ (Shock, CHF or reinfarction upto 30 days). Our study also demonstrates the significance of early thrombolysis.

In this study, the overall rate of CST by tenecteplase was 92\%. Similar results were also observed in Indian registry, where more than $90 \%$ of CST was seen with tenecteplase including high risk subgroup. ${ }^{4}$ In earlier studies, the incidence of diabetes in acute myocardial infarction is $10 \%$ $25 \%$. In our study the prevalence was much higher, $49 \%$. Similarly, high rate of diabetes was mentioned in a study by Satya Murthy et al, $44.94 \% .^{6}$ 


\section{Jemds.com}

ST segment resolution, a useful indicator of coronary reperfusion, is valuable in evaluating the success of myocardial in reperfusion. In this study, overall STE resolution was $94 \%$ and at 90 minutes STE resolution was $50 \%$. Coronary pain is the evidence of ongoing ischaemia or myocardial necrosis. In our study, $92 \%$ had chest pain relief with tenecteplase therapy.

Inpatient mortality rate was low, $3 \%$ compared to CREATE registry (8.6\%). ${ }^{7}$ The mortality rate in ASSENT-2 is upto $6.18 \%$ and upto $6.5 \%$ in TIMI-10 B study.8,9 Indian registry reported a total mortality of $1.69 \%$. The incidence of bleeding is $4 \%$ and stroke (without haemorrhage) is $1 \%$. In ASSENT-2 trial study, the incidence of bleeding is $4.6 \%$.

\section{CONCLUSION}

Thrombolytic therapy is an evidence-based treatment for STEMI. Our study further reinforces the safety and efficacy of tenecteplase in Indian STEMI patients. Our study reconfirms the importance of early thrombolysis for successful thrombolysis, especially in Indian scenario where reaching PCI centre may not be immediately possible.

\section{REFERENCES}

[1] Xavier D, Pais P, Devereaux PJ, et al. Treatment and outcomes of acute coronary syndromes in India (CREATE): a prospective analysis of registry data. Lanset 2008;371(9622):1435-42.

[2] Noorani F, Runge M, Tripathi S, et al. Pre-Hospital delays in care for STEMI patients in Mumbai: challenges and opportunities. Abstract 14400. Circulation 2016;134:A14400.

\section{Original Research Article}

[3] Saran RK, Sethi R, Nagori M. Tenecteplase--the best among the equals. Indian Heart J 2009;61(5):454-8.

[4] Iyengar SS, Nair T, Hiremath JS, et al. Pharmacologic reperfusion therapy with indigenous tenecteplase in 15,222 patients with ST elevation myocardial infarction - the Indian registry. Indian Heart J 2013;65(4):436-41.

[5] Armstrong PW, Gershlick AH, Goldstein P, et al. Fibrinolysis of primary PCI in ST-segment elevation myocardial infarction. $\mathrm{N}$ Engl $\mathrm{J}$ Med 2013;368(15):1379-87.

[6] Sathyamurthy I, Jayanthi K, Iyengar SS, et al. Efficacy and safety of tenecteplase in diabetic and non-diabetic patients of STEMI - Indian registry data. J Assoc Physicians India 2010;58:229-30.

[7] Mohanan PP, Mathew R, Harikrishnan S, et al. Presentation, management and outcomes of 25,748 acute coronary syndrome admissions in Kerala, India: results from the Kerala ACS Registry. Eur Heart J 2013;34(2):121-9.

[8] Cannon CP, Gibson CM, McCabe CH, et al. TNK-tissue plasminogen activator compared with front - loaded alteplase in acute myocardial infarction: results of the TIMI 10B trial. Thrombolysis in Myocardial Infarction (TIMI) 10B Investigators. Circulation 1998;98(25):2805-14.

[9] Assessment of the Safety and Efficacy of a New Thrombolytic (ASSENT - 2) Investigatiors, Van De Werf F, Adgey J, et al. Single-bolus tenecteplase compared with front-loaded alteplase in acute myocardial infarction: the ASSENT-2 double-blind randomized trial. Lancet 1999;354(9180):716-22. 